\title{
Control Network Generator For Latency Insensitive Designs
}

\author{
Eliyah Kilada, Kenneth S. Stevens \\ University of Utah \\ Eliyah.Kilada@utah.edu, kstevens@ece.utah.edu
}

\begin{abstract}
Creating latency insensitive or asynchronous designs from clocked designs has potential benefits of increased modularity and robustness to variations. Several transformations have been suggested in the literature and each of these require a handshake control network (examples include synchronous elasticization and desynchronization). Numerous implementations of the control network are possible. This paper reports on an algorithm that has been proven to generate an optimal control network consisting of the minimum number of 2-input join and 2-output fork control components. This can substantially reduce the area and power consumption of a system. The algorithm has been implemented in a CAD tool, called CNG. It has been applied to the MiniMIPS processor showing a $14 \%$ reduction in the number of control steering units over a hand optimized design in a contemporary work.
\end{abstract}

\section{INTRODUCTION}

A great attention has been recently given to latency-insensitive (LI) designs. LI designs, among other benefits, allow for correct operation independently of channel latencies [1]. This, in turn, facilitates handling any channel delay variations (e.g., due to routing or technology migration), that are typically hard to estimate until the layout in the target technology is complete. This is done by increasing the channel latency without affecting the whole system functionality. This change, for non-LI designs, would require severe manual changes in the system to accommodate the new delays and, possibly, a number of iterations [2], [3]. Synchronous elasticization [2], [4], [5] and desynchronization [6], [7] have been recognized as two promising approaches of transforming an ordinary clocked system into an LI one. A typical first step in both of these approaches is to replace each flip flop in the original design with an LI synchronizing module with a controller [8]. Following this step, communications among registers of the original design are analyzed (i.e., for each register, which registers do send and receive data to and from it). For each register-to-register data communication there must be a corresponding LI control channel, to control data flow between these two registers. This forms a network of control channels. Joins and forks are used to join control channels targeting the same destination registers, and to fork control channels originating from the same source registers, respectively.

The control network can be constructed in many different ways. This paper provides an algorithm, and a CAD tool, that automatically generates a control network with minimum total number of 2-input joins and 2-output forks. This can substantially reduce power and area in the control network. The algorithm has been also applied to the MiniMIPS processor showing a 14\% reduction in the number of control steering units over a hand optimized design in a contemporary work [4].

\section{Problem Definition}

Example 1. Let $A, B, C, D$ be four registers in the original ordinary clocked design. Both registers $A$ and $B$ pass data to both registers $C$ and D. Find a control network implementation for the $L I$ version of this design.

Figures 1a and $1 \mathrm{~b}$ are two example implementations for such a control network. The control network in Fig. 1b has one less

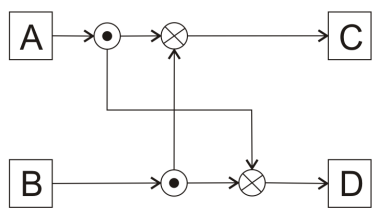

(a)

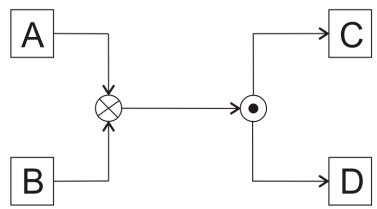

(b)
Fig. 1. Two possible implementations of Example 1. Fork and join components are represented by $\odot$ and $\otimes$, respectively.

join and one less fork component than the network of Fig. 1a. Things get more complicated when the number of registers and their corresponding communications increase. Hence, the purpose of the proposed algorithm is, given a set of required register-toregister communications, the algorithm should automatically generate a control network with minimum total number of 2-input join and 2-output fork components.

In this section we list number of definitions required to formalize the problem. Example 2 will be used as a running example throughout the paper.

Example 2. Let $A, B, C, D, E, F, G, X_{1}, X_{2}, X_{3}, X_{4}, X_{5}$ be twelve registers in the original ordinary clocked design. The following registers pass data to $X_{1}: B, C, G$, and to $X_{2}: A, B, C, G$, and to $X_{3}: A, B, C, D, E$, and to $X_{4}: A, B, D, E, F$, and to $X_{5}: A, B, E, F$. Find a control network implementation for the $L I$ version of this design, that incorporates minimum number of joins and forks components.

In a register-to-register communication, we call the data transmitting register, a source, and the data receiving register, a destination. In Example 2, the following registers are sources: $A, B, C, D, E, F, G$, and the following are destinations: $X_{1}, X_{2}, X_{3}, X_{4}, X_{5}$. The vector of all sources and the vector of all destinations in the network are designated as SourceS and DestinationS, respectively. A component joining two or more control channels into one channel is called a join. A component forking a control channel into two or more control channels is called a fork. Throughout this paper we assume that n-input (n-output) join (fork) is implemented by concatenating $(n-1)$ 2-input (2-output) join (fork) components. 2input join and 2-output fork components will be designated as Join2 (or J2) and Fork2 (or F2), respectively.

Definition 1. Term A set of one or more source registers.

Constructing a term typically means joining the control channels coming from these source registers into one control channel. Each term has a unique identifier, TermID. As an example, a term that joins the control channels coming from: $B, D, E$, is $\{B, D, E\}$ and, for simplicity, will be referred to as $B D E$. $\mid$ Term $_{1} \mid$ designates the cardinality of Term ${ }_{1}$. A term that is composed of only one source register, is called single letter term or SLTerm. The vector of all single 
letter terms (or all source registers) is designated as SLTermS.

Definition 2. Target A term that is associated with a destination register. A target of a certain destination register is a term composed of all source registers that send data to that destination.

In Example 2, $B C G$ is the target term associated with $X_{1}$. The vector of all target terms is designated as TargetS.

Definition 3. Common term or CTerm A term that is the intersection of two or more targets. Formally, a term, CTerm 1 is a CTerm iff CTerm $_{1}=\bigcap_{i=1}^{n}$ Target $_{i}$ for any $1<n \leq \mid$ Target $S \mid$.

Cardinality of a $C$ Term must be greater than one. In Example 2, Term $_{1}=B C G$ would be a $C T e r m$ since it is the intersection of the target terms associated with $X_{1}$ (i.e., $B C G$ ) and $X_{2}$ (i.e., $A B C G$ ). We define $C T$ erm $S$ to be the vector of all common terms, ordered by their cardinalities from the largest to the smallest.

Definition 4. Potential terms or PTermS A vector composed of the concatenation of CTermS and SLTermS, respectively.

Finally, TermS is the concatenation of TargetS and PTermS, respectively. TermS of Example 2 is listed in Table I.

Definition 5. Partial Solution or PS A set of terms that could be used to implement a certain term. Formally, $P S_{t}$ is a partial solution

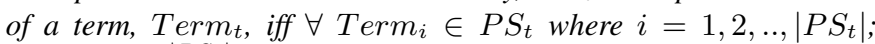
Term $_{t}=\bigcup_{i=1}^{\left|P S_{t}\right|}$ Term $_{i}$

$P S_{t}$ represents one way of constructing $\mathrm{Term}_{t}$. One term could be constructed in multiple ways, and thus has more than one $P S$. In Example 2, to construct Term Te $_{t} A B C D E$, one possible $P S$, is $\{A B C, D, E\}$. Another, is $\{A B D E, C\}$.

Definition 6. Solution or Soln A vector of $P S$ 's, where TermID's are used as indices. If $S_{\text {oln }}$ is a solution, and Term $I D$ is the TermID of Term, then Soln ${ }_{1}\left[\right.$ Term $\left._{t} I D\right]$ is the chosen PS to construct Term in Soln $_{1}$.

For brevity, $\operatorname{Soln}_{1}\left[\operatorname{Term}_{t} I D\right]$ and $\operatorname{Soln}_{1}\left[\right.$ Term $\left._{t}\right]$ will be used interchangeably. In Example 2, the following is a possible solution (Terms are sorted by their TermID's of Table I, and SLTerms $P S$ 's are ignored):

$$
\begin{aligned}
\text { Soln }_{1}= & <\{B C G\},\{B C G, A\},\{A B D E, C\},\{A B D E, F\} \\
& \{A B E F\},\{A B E, D\},\{A B E, F\},\{B C, G\} \\
& \{A B, C\},\{A B, E\},\{B, C\},\{A, B\}>
\end{aligned}
$$

Hence, solution, $S o l n_{1}$, can be seen as a vector of $P S$ choices of the different terms used in Soln 1 . For example, Soln 1 [2] = $\{B C G, A\}$. This means the $P S=\{B C G, A\}$ is used in $S_{0} \ln _{1}$ to construct term $A B C G$ (whose TermID is 2). $S o l n_{1}$ is depicted in Fig. 2.

Definition 7. nUsed $n U$ sed $\left[\right.$ Term $\left._{i}\right]$ defines how many times Term Ter $_{i}$ is used to construct other useful terms in a certain solution. Formally, $n U$ sed $\left[\right.$ Term $\left._{i}\right]$ in a solution, Soln $n_{1}$, is defined recursively to be the number of terms, Term, that satisfy the following two conditions:

1) Term $_{i} \in P S_{t}$ where Soln $_{1}\left[\right.$ Term $\left._{t}\right]=P S_{t}$.

2) $n U$ sed $\left[\right.$ Term $\left._{t}\right]>0$ in Soln $_{1}$. $n U$ sed of all targets are 1 .

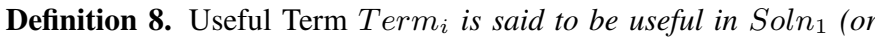
Soln $_{1}$ uses Term $_{i}$ ), iff $n U$ sed $\left[\right.$ Term $\left._{i}\right]>0$ in Soln $_{1}$.

For Example 2 and $S_{0} n_{1}$ of Eq. 1: term ABE (with TermID of 10 ) is used to construct both terms $A B D E$ (with TermID of 6) and

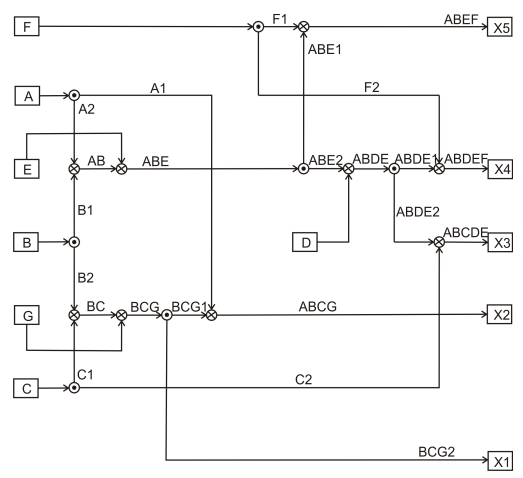

Fig. 2. A sample control network of Example 2.

$A B E F$ (with TermID of 7). Hence, $n U \operatorname{sed}[A B E]$ in $\operatorname{Soln}_{1}$ is 2 . Also, term $A B C$ (with TermID of 9) is not useful in $S_{\text {oln }}$. Term $A B$ (with TermID of 12) is used to construct both terms $A B C$ (with TermID of 9) and $A B E$ (with TermID of 10). However, since term $A B C$ is not useful in $\operatorname{Soln}_{1}$, therefore, $n U \operatorname{sed}[A B]$ in $S_{\text {oln }}$ is only 1 .

Definition 9. Usable Term Usability is defined recursively as follows: Term $_{i}$ is usable iff it belongs to, at least, one PS of one usable term. All targets are usable.

In other words, a term is usable iff it is useful in at least one solution. A term can be usable, but not necessarily useful in some solutions.

Definition 10. Cost $A$ function that returns the number of 2-input joins (J2's) required to implement a term or a Soln.

Formally, let $P S_{t}$ be the $P S$ of term, Term ${ }_{t}$, in $S o l n, S o l n_{1}$, then $\operatorname{Cost}\left(\operatorname{Term}_{t}\right)$ in $\operatorname{Soln}_{1}$ is defined as follows:

$$
\operatorname{Cost}\left(\text { Term }_{t}\right)=\left|P S_{t}\right|-1+\sum_{i=1}^{\left|P S_{t}\right|} \frac{\operatorname{Cost}\left(\text { Term }_{i}\right)}{n U \operatorname{sed}\left[\text { Term }_{i}\right]}
$$

where $\forall i=1,2, . .\left|P S_{t}\right|$, Term $_{i} \in P S_{t} . \operatorname{Cost}\left(\right.$ Term $\left._{t}\right)$ in $\operatorname{Soln}_{1}$ and $\operatorname{Cost}\left(P S_{t}\right)$ in $\operatorname{Soln}_{1}$ will be used interchangeably (since $\operatorname{Soln}_{1}\left[\right.$ Term $\left.\left._{t}\right]=P S_{t}\right)$. Two factors contribute to $\operatorname{Cost}\left(\right.$ Term $\left._{t}\right)$. First, the number of $J 2$ 's used to join $P S_{t}$ 's constituent terms. We assume, in Eq. 2, that, to implement an $n$-input join, $(n-1) J 2$ 's are required. The other factor is the cost of the constituent terms themselves. $\operatorname{Cost}\left(\right.$ Term $\left._{t}\right)$, in a solution, takes into account how much the constituent terms are shared among other terms in that solution. This information is provided by $n U$ sed vector. Cost of all SLTerms are zero.

For Example 2 and $\operatorname{Soln}_{1}$ of Eq. 1: the chosen $P S$ to construct term $A B E$ (with TermID of 10 ) is $\{A B, E\}, n U \operatorname{sed}[A B]=1$. Hence, $\operatorname{Cost}(A B E)=1+\operatorname{Cost}(A B)$. The chosen $P S$ to construct term $A B$ (with TermID of 12) is $\{A, B\}$, and, hence, $\operatorname{Cost}(A B)=1$. Therefore, $\operatorname{Cost}(A B E)$ in $\operatorname{Soln}_{1}$ is 2. Similarly, $\operatorname{Cost}(A B D E)=2$.

Similarly, we also define $\operatorname{Cost}\left(\operatorname{Soln}_{1}\right)$ to be the total number of $J 2$ 's used to construct all the targets in $S_{0} n_{1}$. Formally,

$$
\operatorname{Cost}\left(\operatorname{Soln}_{1}\right)=\sum_{i=1}^{\mid \text {Target } S \mid} \operatorname{Cost}\left(\text { Target }_{i}\right)
$$

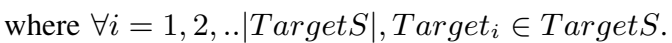

For Example 2 and $S_{\text {oln }}$ of Eq. 1: we have five targets (those that are associated with the five destinations), namely, $B C G, A B C G$, 
$A B C D E, A B D E F, A B E F$. The summation of the costs of these targets in $\operatorname{Soln}_{1}$ (i.e., $\operatorname{Cost}\left(\operatorname{Soln}_{1}\right)$ is 9.

Definition 11. Optimum Solution or OptSoln A solution with minimum Cost among all other solutions.

Definition 12. $\mathrm{nAJ}(\mathrm{Term})$ If $P S_{t}$ is the chosen PS of Term in $S_{\text {oln }}$, then, $n A J\left(\right.$ Term $\left._{t}\right)$ is the number of J2's added to the

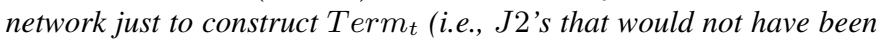

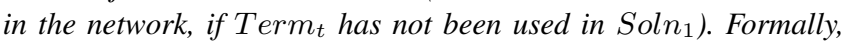

$$
n A J\left(\text { Term }_{t}\right)=\left|P S_{t}\right|-1+\sum_{i=1}^{\left|P S_{t}\right|} n A J\left(\text { Term }_{i}\right) \times s_{i}
$$

where $\forall i=1,2, . .\left|P S_{t}\right|$, Term $_{i} \in P S_{t}$ and $s_{i}=1$ if $n U \operatorname{sed}\left[\right.$ Term $\left._{i}\right]=1$ and $s_{i}=0$ if $n U \operatorname{sed}\left[\right.$ Term $\left._{i}\right]>1$

$n A J\left(\right.$ Term $\left._{t}\right)$ in $\operatorname{Soln}_{1}$ and $n A J\left(P S_{t}\right)$ in $\operatorname{Soln}_{1}$ will be used interchangeably (since $\operatorname{Soln}_{1}\left[\right.$ Term $\left._{t}\right]=P S_{t}$ ). As an example, let all the terms used by $P S_{t}$ be already shared by other terms in $S o l n_{1}$. In this case, all that is added to the network to construct $P S_{t}$ is the $J 2$ 's required to join its constituent terms (i.e., $\left|P S_{t}\right|-1$ ).

For Example 2 and $\operatorname{Soln}_{1}$ of Eq. 1: $n A J(A B)=1$, $n A J(A B E)=2, n U \operatorname{sed}[A B E]=2$. Although the Cost of $A B D E$ is 2 , its $n A J$ is only 1 . The reason is, term $A B E$ which is used to construct $A B D E$ in $\operatorname{Soln}_{1}$ is also used in the solution to construct another term (i.e., term $A B E F$ with TermID of 7). Hence, to construct term $A B D E$, the only added $J 2$ to $S_{o l n}$ is the join required to join $A B E$ with $D$.

\section{ALGORITHM}

Lemma 1. If $n J 2$ and $n F 2$ are the total number of J2's and $F 2$ 's in the network, respectively. Then, whatever the PS choices of different terms used in a solution, the following equality holds: $n J 2-n F 2=\mid$ Source $S|-|$ Destination $S \mid$

Proof: Proof is omitted due to space limitation.

Theorem 2. An algorithm that minimizes $n J 2$ will also minimize $n F 2$ and also $n J 2+n F 2$.

In other words, OptSoln defined in Def. 11 will incorporate minimum total number of J2's and F2's in the network.

Proof: The theorem follows directly from Lemma 1.

Theorem 2 narrows down the problem to: Given a set of source registers and a set of targets, the algorithm aims to construct the targets by using minimum total number of $J 2$ 's (i.e., to find OptSoln). The proposed algorithm consists of four main steps, covered in the following four subsections. The first step (covered in Subsection III-A) is to find the candidate terms that can be used in OptSoln. Then, for each of the candidate terms, the algorithm finds the candidate $P S$ 's that may be used by OptSoln. This step is explained in Subsection III-B. At this point the search space of the problem consists of all the possible $P S$ choices of all the candidate terms. In step 3 (covered in Subsection III-C), the algorithm does a number of iterations. At the end of each iteration, it eliminates part of the search space. When the algorithm can do no more elimination, it goes to step 4. Step 4 (covered in Subsection III-D) does a final possible reduction in the remaining search space. It then calculates the Cost of the remaining solutions and returns OptSoln.

\section{A. Construct Potential Terms}

Theorem 3. (Potential Terms) An optimum solution can be found by using only potential terms (or PTermS, Def. 4).
Proof: Proof is omitted due to space limitation.

The first step in the algorithm is to determine which terms could be used to construct the targets. It tries to exclude terms that are guaranteed not to be used in OptSoln. Theorem 3 narrows down the search space, by confining the candidate terms to PTermS. PTerm $S$ are constructed following their definition in Def. 4.

\section{B. Construct Partial Solutions}

The search space (i.e, the possible solutions), at this point, consists of all combinations of all possible $P S$ choices of all PtermS. This step aims at excluding $P S$ 's that will not be needed in OptSoln. A cost metric must be used that can differentiate between two $P S$ 's of a certain term without actual search space exploration. Following are a set of theorems that guide this step.

Theorem 4. (nAJ(PS)) Let Soln 1 and Soln 2 be two solutions. Let also, $\forall(i=1,2, . . \mid$ Term $S \mid \wedge i \neq t)$ : $\operatorname{Soln}_{1}\left[\right.$ Term $\left._{i}\right]=$ $\operatorname{Soln}_{2}\left[\right.$ Term $\left._{i}\right]$, Soln $_{1}\left[\right.$ Term $\left._{t}\right]=P S_{t 1}$ and Soln STerm $\left._{t}\right]=$ $P S_{t 2}$. Then, if $\left(\left(n A J\left(P S_{t 1}\right)\right.\right.$ in $\left.S_{0 l n}\right) \geq\left(n A J\left(P S_{t 2}\right)\right.$ in $\left.\left.S_{0 l n}\right)\right)$, then $\operatorname{Cost}\left(\operatorname{Soln}_{1}\right) \geq \operatorname{Cost}\left(\operatorname{Soln}_{2}\right)$. Greater and equal operators are ordered respectively.

Proof: Proof is omitted due to space limitation.

Corollary 5. Let $P S_{1}$ and $P S_{2}$ be two $P S$ 's of Term. Then, if, for all possible combinations of other terms' $P S$ 's choices, $n A J\left(P S_{1}\right)>n A J\left(P S_{2}\right)$ then OptSoln will not use PS

Corollary 6. Let $P S_{1}$ and $P S_{2}$ be two PS's of Term. Then, if, for all possible combinations of other terms' $P S$ 's choices, $n A J\left(P S_{1}\right) \geq n A J\left(P S_{2}\right)$ then OptSoln can be found that doesn't use $P S_{1}$.

Proof of both Corollaries 5 and 6 follows from Theorem 4 as well as Definitions 10 and 11.

Theorem 7. (Cost(PS)) Let Soln 1 and Soln 2 be two solutions. Let also, $\forall(i=1,2, . . \mid$ Term $S \mid \wedge i \neq t)$ : $\operatorname{Soln}_{1}\left[\right.$ Term $\left._{i}\right]=$

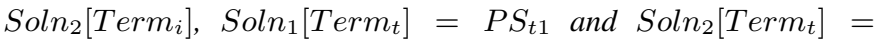
$P S_{t 2}$. Then, if $\left(\left(\operatorname{Cost}\left(P S_{t 1}\right)\right.\right.$ in $\left.\operatorname{Soln}_{1}\right) \geq\left(\operatorname{Cost}\left(P S_{t 2}\right)\right.$ in $\left.\left.S_{\text {oln }}\right)\right)$, then the following inequality does not necessarily hold: $\operatorname{Cost}\left(\operatorname{Soln}_{1}\right) \geq \operatorname{Cost}\left(\operatorname{Soln}_{2}\right)$.

Proof: Proof and counter example are omitted due to space limitation.

Based on theorems 4 through 7, nAJ (rather than Cost) could be used to differentiate between two $P S$ 's of a certain term without actual search space exploration. The following three theorems, set a list of rules that should be considered while constructing the $P S$ 's of the terms in PTermS.

Theorem 8. (Rule 1) Adding a whole redundant term to a PS always causes it to be more expensive (in terms of nAJ). Formally,

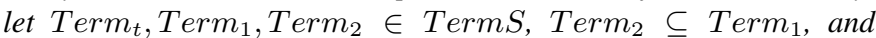
Term $_{1} \subseteq$ Term $_{t}$. Let $P S_{1}$ and $P S_{2}$ be two PS's of Term. Let both $P S_{1}$ and $P S_{2}$ be the same except that $P S_{1}$ contains one Term $_{1}$, while P $S_{2}$ contains one Term ${ }_{1}$ and one Term ${ }_{2}$. Then, an optimum solution will not use $\mathrm{PS}_{2}$.

Proof: Proof is omitted due to space limitation.

Consider term $A B C G$ in Example 2. $P S_{1}=\{A, B C G\}$ is always cheaper than $P S_{2}=\{A, B C G, B C\}$. Hence, $P S_{2}$ should be excluded from the search space.

Theorem 9. (Rule 2) While searching for OptSoln, using a term in a $P S$ is always the same or cheaper (in terms of $n A J$ ) than using all its 
TABLE I

TERMS AND $P S$ 'S OF EXAMPLE 2

\begin{tabular}{|c|c|c|c|c|c|c|}
\hline TermID & Term & Type & PSID & PS & \multicolumn{2}{|c|}{$\begin{array}{l}\text { Initial nUsed } \\
\text { Max }\end{array}$} \\
\hline 1 & $B C G$ & $T$ & 1 & $\{B C G\}$ & 1 & 1 \\
\hline 2 & $A B C G$ & $T$ & 1 & $\begin{array}{c}\{B C G, A\} \\
\{A B C, G\}\end{array}$ & 1 & 1 \\
\hline 3 & $A B C D E$ & $T$ & 1 & $\begin{array}{c}\{A B D E, C\} \\
\{A B C, D, E\}\end{array}$ & 1 & 1 \\
\hline 4 & $A B D E F$ & $T$ & 1 & $\begin{array}{c}\{A B D E, F\} \\
\{A B E F, D\}\end{array}$ & 1 & 1 \\
\hline 5 & $A B E F$ & $T$ & 1 & $\{A B E F\}$ & 1 & 1 \\
\hline 6 & $A B D E$ & $C, P$ & 1 & $\{A B E, D\}$ & 2 & 0 \\
\hline 7 & $A B E F$ & $C, P$ & 1 & $\{A B E, F\}$ & 2 & 1 \\
\hline 8 & $B C G$ & $C, P$ & 1 & $\{B C, G\}$ & 2 & 1 \\
\hline 9 & $A B C$ & $C, P$ & 1 & $\{B C, A\}$ & 2 & 0 \\
\hline 10 & $A B E$ & $C, P$ & 1 & $\{A B, C\}$ & & \\
\hline 11 & $B C$ & $C, P$ & 1 & $\{B, C\}$ & 2 & 1 \\
\hline 12 & $A B$ & $C, P$ & 1 & $\{A, B\}$ & 2 & 1 \\
\hline $13-19$ & $A-G$ & $S, P$ & 1 & & & \\
\hline
\end{tabular}

constituent terms. Formally, let Term ${ }_{t}$, Term $_{1}$, Term $_{2}$, Term $_{3} \in$ TermS, Term $1 \subseteq$ Term $_{t}$, and Term TeT $_{1}=$ Term $_{2} \cup$ Term $_{3}$. Let

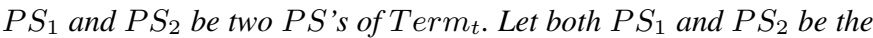
same except that $P S_{1}$ contains one Term $m_{1}$, while $P S_{2}$ contains one Term $_{2}$ and one Term 3 . Then, an optimum solution can be found that doesn't use $P S_{2}$.

Proof: Proof is omitted due to space limitation.

Consider term $A B C G$ in Example 2. While searching for OptSoln, $P S_{1}=\{A, B C G\}$ is always the same or cheaper than $P S_{2}=\{A, B C, G\}$. Hence, $P S_{2}$ could be excluded from the search space.

Theorem 10. (Rule 3) An SLTerm is always the same or cheaper (in terms of nAJ) than any other non - SLTerm. Let Term Term $_{1}$, Term $_{3} \in$ TermS and $\notin S L T e r m S$, and $S_{\text {LTerm }} \in$ SLTermS. Let also Term $1, S L T e r m_{2}$, Term $_{3} \subseteq$

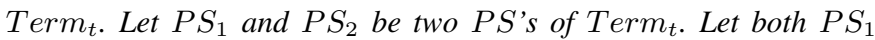
and $P S_{2}$ be the same except that $P S_{1}$ contains one $S L T e r m_{2}$,

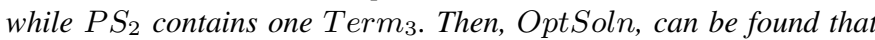
doesn't use $P S_{2}$.

Proof: Proof is omitted due to space limitation.

Consider term $A B C G$ in Example 2. $P S_{1}=\{B C G, A\}$ is always the same or cheaper than $P S_{2}=\{B C G, A B\}$. Hence $P S_{2}$ should be excluded from the search space.

An algorithm has been developed, that takes into account all the three rules while constructing $P S$ 's of the terms. The algorithm has been omitted due to space limitation. Table I shows the $P S$ 's of Example 2 after applying the three rules.

\section{Update Cost Structures And Remove Higher nAJ Partial Solutions}

Theorem 3 narrowed down the search space by confining the number of candidate terms. Furthermore, Theorems 4 through 10 reduced their possible corresponding $P S$ 's. At this point the search space of the problem consists of all the remaining possible $P S$ choices of all the candidate terms. This step aims at pruning out the search space, even more, through a number of iterations. At the end of each iteration, more information about the cost of the different possible solutions, as well as OptSoln, is revealed and, hence, more

${ }^{1} \mathrm{~T}$ is for Target, $C$ for CTerm, $P$ for PTerm and $S$ for SLTerm. areas of the search space can be eliminated. When the algorithm can do no more eliminations, it goes to the next step.

Although, the value of $n A J\left(\right.$ Term $\left._{t}\right)$ in a certain solution, $S o l n_{1}$, (or, equivalently, the value of $n A J\left(P S_{t}\right)$ in $S o l n_{1}$, where $\operatorname{Soln}_{1}\left[\right.$ Term $\left.\left._{t}\right]=P S_{t}\right)$, depends on the $P S$ 's choices of the other terms (which we call, hereafter, the environment). And, the search space, at this point, allows for all $P S$ 's choices of the environment - which allows $n A J\left(P S_{t}\right)$ to take numerous values. However, we can still utilize some information we know about the environment to deterministically differentiate between two $P S$ 's of a certain term based on their $n A J$. The type of information needed is supplied by $n U$ sedMax and $n U$ sedMin which are defined, along with other related concepts, as follows:

Definition 13. nUsedMax $A$ vector of numbers, where TermID's are used as indices. $n U$ sedMax $\left[\right.$ Term $\left._{i}\right]$ is the number of usable

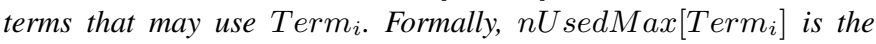
number of Term ${ }_{t}$ 's that satisfy the following two conditions:

1) Term $_{i} \in P S_{t}$ where PS $S_{t}$ is a PS of Term . $_{\text {. }}$

2) Term $_{t}$ is usable.

$n U$ sedMax $\left._{\text {Target }}\right]=1 \forall$ Target $_{i} \in$ Target $_{\text {. }}$

Table I shows the initial values of $n U$ sedMax of different terms in Example 2. At the end of each iteration, some $P S$ 's are omitted from the search space, and, hence, the value of $n U$ sedMax of some terms will be decreased.

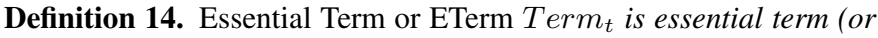
ETerm) iff it is useful in OptSoln.

All targets are ETerms.

Definition 15. Essential Child or EChild Term T $_{i}$ is said to be an

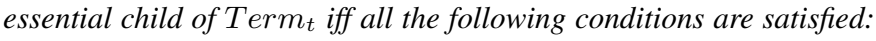

1) Termi belongs to all PS's of Term . $^{\text {. }}$.

2) Term $_{t}$ is usable.

We also define EChildren $\left[\right.$ Term $\left._{t}\right]$ to be all EChild terms of Term $_{t}$.

Definition 16. nUsedMin A vector of numbers, where TermID's are used as indices. nUsedMin $\left[\right.$ Term $\left._{i}\right]$ is the number of useful terms in OptSoln that are guaranteed to use Termi. Formally, $n U$ sedMin $\left[\right.$ Term $\left._{i}\right]$ is the number of Term ${ }_{t}$ 's that satisfy the following two conditions:

1) Term $_{i} \in P S_{t}$ where OptSoln $\left[\right.$ Term $\left._{t}\right]=P S_{t}$.

2) Term $_{t}$ is useful in OptSoln.

$n U$ sedMin $\left._{\text {Target }}\right]=1 \forall$ Target $_{i} \in$ Target $S_{\text {. }}$

The calculation of $n$ UsedMin is initialized by having

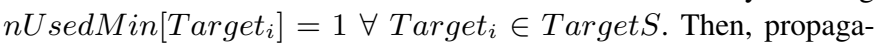

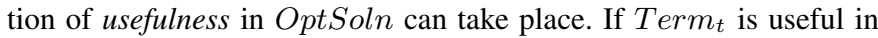
OptSoln, then all its EChildren will also be useful in OptSoln. An algorithm that automates this process has been omitted due to space limitation.

Table I shows the initial values of $n U$ sedMin of different terms in Example 2. At the end of each iteration, more information about OptSoln are revealed, and, hence, the value of $n U$ sedMin of some terms will be increased.

Definition 17. nAJMax(PS) nAJMax $\left(P S_{t}\right)$ is the value of $n A J\left(P S_{t}\right)$ when the environment provides minimum sharing to the terms used by $P S_{t}$. Formally, let $P S_{t}$ be a $P S$ of Term , then 
$n A J M a x\left(P S_{t}\right)$ is defined as follows:

$$
\begin{aligned}
n A J \operatorname{Max}\left(P S_{t}\right)= & \left|P S_{t}\right|-1+ \\
& \sum_{i=1}^{\left|P S_{t}\right|} n A J M a x\left(\text { Term }_{i}\right) \times s_{i}
\end{aligned}
$$

where $\forall i=1,2, . .\left|P S_{t}\right|$, Term $_{i} \in P S_{t}$ and $s_{i}=1$ if $x_{i}=1$ and $s_{i}=0$ if $x_{i}>1$, where $x_{i}=1+$ the number of Term 1 's (where Term $_{t 1} \neq$ Term $_{t}$ ) that satisfy the following two conditions: First, Term TeP $_{t 1}$ where OptSoln $\left[\right.$ Term $\left._{t 1}\right]=P S_{t 1}$. Second, Term $_{t 1}$ is useful in OptSoln.

It is a trivial task to compute $x_{i}$ in Def. 17 from $n U \operatorname{sedMin}$.

Definition 18. nAJMin(PS) $n A J M i n\left(P S_{t}\right)$ is the value of $n A J\left(P S_{t}\right)$ when the environment provides maximum sharing to the terms used by $P S_{t}$. Formally, let $P S_{t}$ be a PS of Termt, then $n A J \operatorname{Min}\left(P S_{t}\right)$ is defined as follows:

$$
\begin{aligned}
n A J \operatorname{Min}\left(P S_{t}\right)= & \left|P S_{t}\right|-1+ \\
& \sum_{i=1}^{\left|P S_{t}\right|} n A J \operatorname{Min}\left(\text { Term }_{i}\right) \times s_{i}
\end{aligned}
$$

where $s_{i}=1$ if $n U \operatorname{sedMax}\left[\right.$ Term $\left._{i}\right]=1$ and $s_{i}=0$ if $n U$ sedMax $\left[\right.$ Term $\left._{i}\right]>1$

Definition 19. nAJMax/Min(Term) Let PSs[Term $\left.{ }_{t}\right]$ be a vector of the PS's of Term. Then,

$$
\begin{aligned}
& n A J \operatorname{Max}\left(\operatorname{Term}_{t}\right)=\stackrel{\left|P S s\left[\operatorname{Term}_{t}\right]\right|}{\max _{i=1}} n A J \operatorname{Max}\left(P S_{t i}\right) \\
& n A J \operatorname{Min}\left(\text { Term }_{t}\right)=\stackrel{\mid P S s\left[\text { Term }_{t}\right] \mid}{\min _{i=1}} n \operatorname{AJMin}\left(P S_{t i}\right)
\end{aligned}
$$

A more restricted condition, yet easier to check, of Corollaries 5 and 6 is stated in the following corollaries:

Corollary 11. Let $P S_{1}$ and $P S_{2}$ be two PS's of Termt. Then, if, $n A J \operatorname{Min}\left(P S_{1}\right)>n A J M a x\left(P S_{2}\right)$ then OptSoln will not use $P S_{1}$.

Corollary 12. Let $P S_{1}$ and $P S_{2}$ be two $P S$ 's of Termt. Then, if, $n A J \operatorname{Min}\left(P S_{1}\right) \geq n A J \operatorname{Max}\left(P S_{2}\right)$ then OptSoln can be found that doesn't use $P S_{1}$.

Algorithm 1 makes use of Corollaries 11 and 12, along with $n U$ sedMax and $n U$ sedMin structures to, iteratively, refine the search space. It incorporates the following data structures:

- $n A J M i n / M a x\left[\right.$ Term $\left._{t}\right]$ : A vector that stores $n A J M i n / M a x$ of all $\mathrm{Term}_{t}$, respectively.

- PSnAJMin/Max $\left[\right.$ Term $\left._{t}\right]\left[P S_{t i}\right]$ : A two dimensional structure that stores $n A J M i n / M a x$ of all $P S_{t i}$ of all Term $_{t}$, respectively.

- UT: a set of terms whose nAJMax and/or nAJMin need to be updated. The terms are ordered within the set by their cardinalities starting by the largest to the smallest. UT is initialized with all targets and common terms.

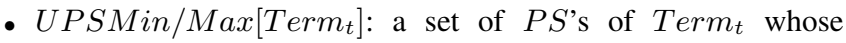
$n A J M i n / M a x$ need to be updated, respectively. They are initialized with all $P S$ 's of Term $_{t}$.

- PSR: a set of $P S$ 's that are scheduled to be removed from the search space.

Algorithm 1 starts with $U T$ initialized with all targets and common terms. Line 2 picks the smallest term in $U T$, Term t $_{\text {. Lines }} 4$ and 5 store the old values of Term,'s nAJMax, nAJMin, EChildren

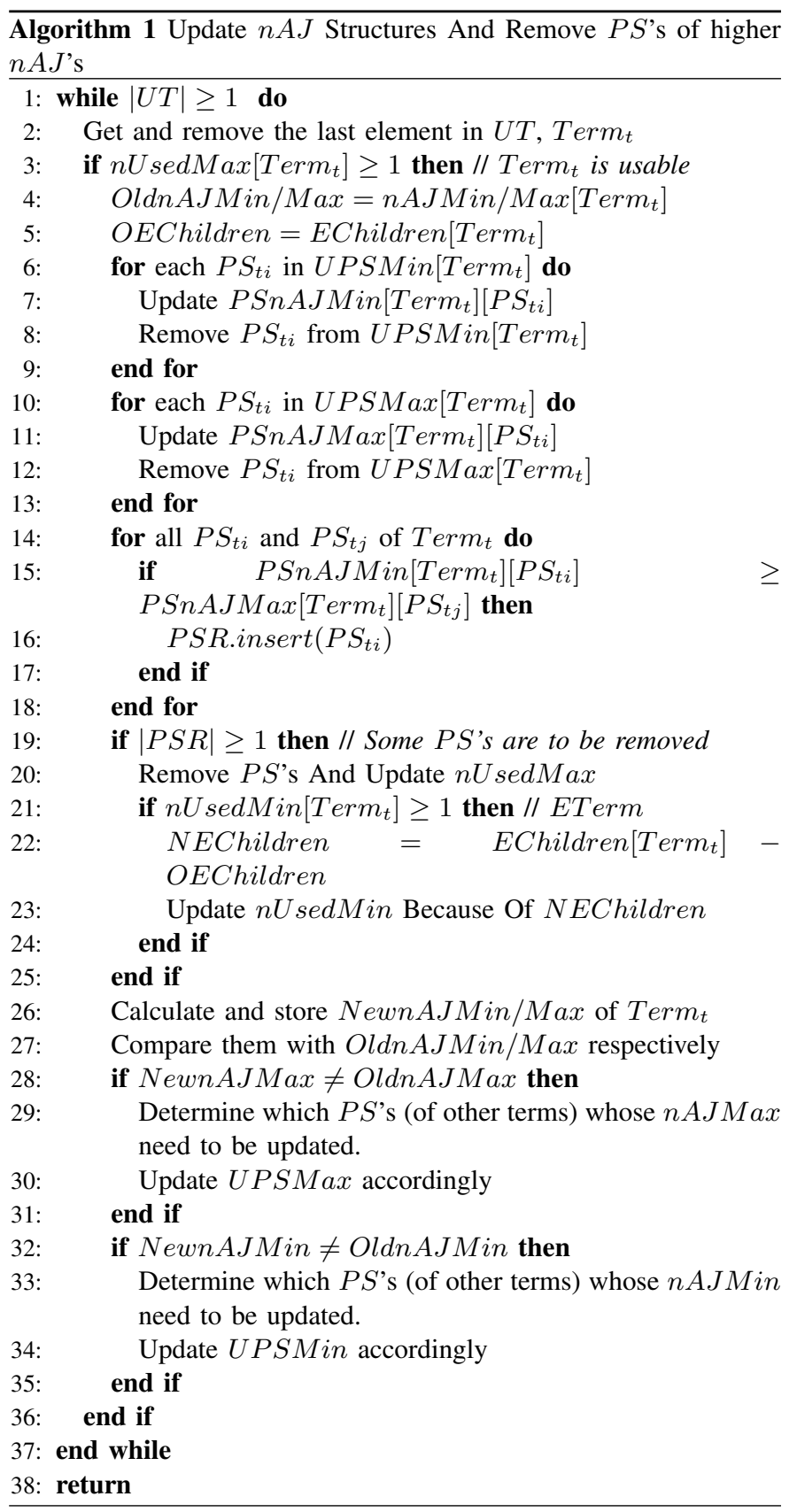

before doing any update. Lines 6 through 9 (Lines 10 through 13) update $n A J \operatorname{Min}(n A J M a x)$ of the PS's of Term specified in UPSMin[Term T $\left._{t}\right]\left(U P S M a x\left[\right.\right.$ Term $\left.\left._{t}\right]\right)$, respectively. Lines 14 through 18 apply Corollary 12 to prune out expensive $P S$ 's. $P S$ 's to be removed are stored in $P S R$. Details of procedure in Line 20 is omitted due to space limitation. That procedure, essentially, propagates the effect of removing a $P S_{t}$ of Term $_{t}$ to $n U$ sedMax of some (or all) of its constituent terms. This, in turn, can affect $n A J M i n$ of some other terms $P S$ 's. The affected terms and $P S$ 's are added to $U T, U P S M i n$, respectively, so that they are updated in the following iterations. Removing $P S$ 's from Term $_{t}$ may not only affect $n U$ sedMax of the constituting terms, but also, may add to EChildren[Term Th $^{\text {. Now, if Term }}$ is an ETerm, and it gained new EChildren in this iteration, then its new EChildren will also become ETerms. This is handled in Lines 21 through 24 


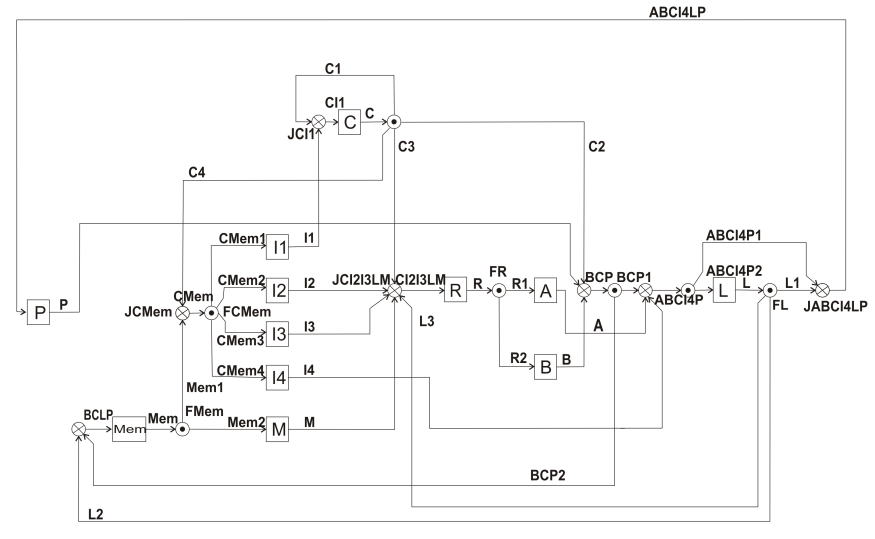

Fig. 3. Control network of the LI version of MiniMIPS processor.

of Algorithm 1. Details of procedure in Line 23 has been omitted due to space limitation. This procedure propagates the effect of essentiality (i.e., $n U \operatorname{sedMin}$ ) to the $n A J M a x$ values of other terms $P S$ 's in the network. Again, the affected terms and $P S$ 's are added to UT,UPSMax, respectively, so that they are updated in the following iterations. Final part of Algorithm 1 (i.e., Lines 26 through 35) checks if there is any change occurred to the values of

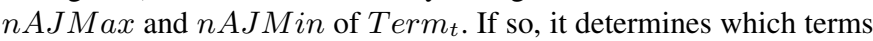
and $P S$ 's are affected by these changes. It changes UT, UPSMax and UPSMin, accordingly. Algorithm 1 will continue to iterate until $U T$ is empty (i.e., no more terms need to be updated).

\section{Find Optimum Solution}

In all the examples included in this paper, the search space, at this point, contains only one solution (i.e., OptSoln). However, in general, we have to explore the remaining search space to find OptSoln. Solutions costs are evaluated using Definitions 7 and 10. Two more lemmas, a theorem, and an algorithm have been developed to expedite finding OptSoln in the remaining search space. However, they have been omitted due to space limitation. In Example 2, OptSoln is the same as $\operatorname{Soln}_{1}$ of Eq. 1, and its Cost is 9.

\section{RESUlTS}

\section{A. Case Study: MiniMIPS}

MIPS (Microprocessor without Interlocked Pipeline Stages) is a 32-bit architecture, first designed by Hennessy [9]. MiniMIPS is an 8-bit subset of MIPS. It is fully described in [10].

MiniMIPS has total of 12 synchronization points (i.e., registers): $P$ (program counter), $C$ (controller), $I 1, I 2, I 3, I 4$ (four instruction registers), $A, B$ and $L$ (ALU two input and one output registers, respectively), $M$ (memory data register), $R$ (register file) and $\mathrm{Mem}$ (memory). The following registers pass data to both $A, B: R$, and to $R: C, I 2, I 3, L, M$, and to $C: C, I 1$, and to $I 1, I 2, I 3, I 4$ : $C, M e m$, and to $L: A, B, C, I 4, P$, and to $M: M e m$, and to Mem : $B, C, L, P$, and to $P: A, B, C, I 4, L, P$.

A hand optimized version of an LI control network of MiniMIPS, is reported in [4]. It incorporates $14 \mathrm{~J} 2$ 's and $14 \mathrm{~F} 2$ 's. On the other hand, applying this algorithm to the same problem, results in the control network of Fig. 3. OptSoln uses 12 J2's and 12 F2's, for a $14 \%$ reduction.

\section{B. CNG Tool}

The algorithm has been coded in C++ within a tool called CNG. CNG accepts an input file with the required register-to-register
TABLE II

CNG RUNTIME OF DIFFERENT PROBLEMS

\begin{tabular}{|c||c|c|c|c|c|}
\hline Problem & $\mid$ SourceS $\mid$ & $\mid$ DestinationS $\mid$ & $\mid$ TermS $\mid$ & Cost & Runtime \\
\hline Example 1 & 2 & 2 & 5 & 1 & $5 \mathrm{~ms}$ \\
\hline Example 2 & 7 & 5 & 19 & 9 & $6 \mathrm{~ms}$ \\
\hline MiniMIPS & 12 & 12 & 29 & 12 & $6 \mathrm{~ms}$ \\
\hline S298 & 17 & 20 & 53 & 22 & $15 \mathrm{~ms}$ \\
\hline
\end{tabular}

communications. It returns OptSoln and the minimum cost. Table II shows CNG runtime of the same examples encountered in this paper, plus two design examples. S298 is an ISCAS-89 benchmark. The machine used has Intel(R) Xeon(TM) CPU 3.20GHz processor and a $2 \mathrm{~GB}$ memory.

\section{CONCLUSION}

Transforming clocked designs into latency insensitive designs require creation of a handshake control network. We presented an algorithm that has been proven to automatically generate an optimal control network consisting of the minimum number of 2-input joins and 2-output forks. We also presented a CAD tool, $\mathrm{CNG}$, that implemented the algorithm. It was applied to the MiniMIPS processor resulting in $14 \%$ reduction in the number of control steering units over a hand optimized design in a contemporary work. The algorithm does not guarantee minimum control network path delay. In some cases, a control path delay can be reduced by reconstructing some common terms rather than sharing them. Additional delays may exist due to the sequential ordering required by sharing. Normally, this is not a problem since the critical data path delay is usually larger than the critical delay in the control network. Adding delay metrics to the algorithm is kept for future work.

\section{ACKNOWLEDGMENT}

This work was supported by the National Science Foundation under grant CCF-0810408.

\section{REFERENCES}

[1] L. Carloni, K. Mcmillan, and A. L. Sangiovanni-VincentelliR, "Theory of latency insensitive design," in IEEE Transactions on CAD of Integrated Circuits and Systems, vol. 20, no. 9, Sep 2001, pp. 1059-1076.

[2] J. Cortadella, M. Kishinevsky, and B. Grundmann, "Synthesis of synchronous elastic architectures," in ACM/IEEE Design Automation Conference, July 2006, pp. 657-662.

[3] L. Carloni and A. Sangiovanni-Vincentelli, "Coping with latency in soc design," Micro, IEEE, vol. 22, no. 5, pp. 24-35, Sep/Oct 2002.

[4] E. Kilada, S. Das, and K. S. Stevens, "Synchronous Elasticization: Practical Considerations And MiniMIPS Case Study," Submitted to The IEEE International Symposium on Circuits and Systems 2010. ISCAS 2010.

[5] H. M. Jacobson, P. N. Kudva, P. Bose, P. W. Cook, S. E. Schuster, E. G. Mercer, and C. J. Myers, "Synchronous interlocked pipelines," in 8th International Symposium on Asynchronous Circuits and Systems, Apr. 2002, pp. 3-12.

[6] J. Cortadella, A. Kondratyev, L. Lavagno, and C. Sotiriou, "Desynchronization: Synthesis of asynchronous circuits from synchronous specifications," Computer-Aided Design of Integrated Circuits and Systems, IEEE Transactions on, vol. 25, no. 10, pp. 1904-1921, Oct. 2006.

[7] N. Andrikos, L. Lavagno, D. Pandini, and C. Sotiriou, "A fullyautomated desynchronization flow for synchronous circuits," in Design Automation Conference, 2007. DAC '07. 44th ACM/IEEE, June 2007, pp. 982-985.

[8] J. Carmona, J. Cortadella, M. Kishinevsky, and A. Taubin, "Elastic circuits," Computer-Aided Design of Integrated Circuits and Systems, IEEE Transactions on, vol. 28, no. 10, pp. 1437-1455, Oct. 2009.

[9] J. H. et al., "The MIPS Machine," in COMPCON, 1982, pp. 2-7.

[10] N. Weste and D. Harris, CMOS VLSI design: a circuit and systems perspective, 2004. 